\title{
TESTES PROJETIVOS NA AVALIAÇÃO PSICOLÓGICA DA ESQUIZOFRENIA: UMA REVISÃO DA LITERATURA
}

\author{
Ana Carolina Zuanazzi \\ Universidade de São Paulo \\ Rafaela Larsen Ribeiro \\ Centro de Diagnóstico Neuropsicológico
}

\begin{abstract}
Resumo
Objetivou-se investigar o que se tem produzido no Brasil sobre a temática do uso de instrumentos projetivos/expressivos na avaliação psicológica de pacientes com esquizofrenia. Para tanto, fez-se uma revisão da literatura brasileira nas bases de dados Scielo, Lilacs, Medline, Pepsic e Index Psicologia a partir dos descritores "avaliação e esquizofrenia" e um dos seguintes termos: Rorschach, TAT, HTP, Pfister, Zulliger, Palográfico ou PMK. Foram selecionados dez artigos a partir dos critérios de inclusão e exclusão. Nos últimos dez anos foram publicados $80 \%$ dos artigos encontrados, sendo o Rorschach o instrumento mais utilizado nesse contexto. Não foram encontrados estudos com os instrumentos HTP, Palográfico, PMK e Zulliger. Apesar do baixo número de estudos na literatura brasileira, considera-se que o uso de instrumentos projetivos na avaliação psicológica de pessoas com esquizofrenia enriquece o diagnóstico e a verificação de potencialidades e dificuldades emocionais e da percepção da realidade nessa população.

Palavras-chave: técnicas projetivas; avaliação psicológica; esquizofrenia; revisão de literatura; teste de Rorschach.

\section{PROJECTIVE TESTS IN PSYCHOLOGICAL ASSESSMENT OF SCHIZOPHRENIA: A REVIEW OF BRAZILIAN LITERATURE}

\begin{abstract}
This article aimed to investigate the Brazilian's production about the use of projective/expressive instruments for psychological assessment in patients with schizophrenia. To do that, we made a review of Brazilian literature in databases Scielo, Lilacs, Medline, Pepsic, and Index Psicologia from descriptors "assessment and schizophrenia" and one of the terms: Rorschach, TAT, HTP, Pfister, Zulliger, Palográfico, or PMK. Ten papers were selected based on inclusion and exclusion criteria. Eight percent of the articles were published in the past decade, and the Rorschach is the most used instrument in this context. No studies were found with tests HTP, Palográfico, PMK, or Zulliger. Despite the reduced number of studies in the Brazilian literature, the use of projective instruments in psychological assessment of schizophrenic enriches the diagnosis and verification of capabilities and emotional difficulties and the perception of reality in this population.
\end{abstract}

Keywords: projective techniques; psychological assessment; schizophrenia; literature review; Rorschach test. 


\title{
PRUEBAS PROYECTIVAS EN LA EVALUACIÓN NEUROPSICOLÓGICA DE LA ESQUIZOFRENIA: UNA REVISIÓN DE LA LITERATURA BRASILEÑA
}

\begin{abstract}
Resumen
Este artículo tuvo como objetivo investigar los cuales han tenido lugar en Brasil en el tema de la utilización de instrumentos proyectivos/expresivos para la evaluación psicológica en pacientes con esquizofrenia. A tal efecto, se realizó una revisión de la literatura brasileña en bases de datos Scielo, Lilacs, Medline, Pepsic y Index Psicologia de descriptores "evaluación y esquizofrenia" y Rorschach, TAT, HTP, Pfister, Zulliger, o Palográfico, o PMK. Se seleccionaron diez artículos desde los criterios de inclusión y exclusión. Durante la década pasada se publicó el $80 \%$ de los artículos encontrados, y el Rorschach el instrumento más utilizado en este contexto. No se encontraron estudios con instrumentos HTP, Palográfico, PMK y Zulliger. Apesar del bajo número de estudios en la literatura brasileña, se considera que el uso de instrumentos proyectivos en la evaluación psicológica de la esquizofrenia enriquece las capacidades de diagnóstico y verificación de dificultades emocionales y la percepción de la realidad en esta población.
\end{abstract}

Palabras clave: técnicas proyectivas; evaluación neuropsicológica; esquizofrenia; revisión de literatura; test de Rorschach.

\section{INTRODUÇÃO}

\section{Esquizofrenia}

A esquizofrenia é classificada como um transtorno grave dentro do grupo das psicoses, de curso crônico e debilitante. Aproximadamente $0,7 \%$ da população geral são acometidas por essa doença (Noto, Ortiz, \& Honda, 2015). É caracterizada por distorções do pensamento e por afeto inadequado ou embotado (Organização Mundial da Saúde [OMS], 1993; Villemor-Amaral et al., 2005). Tais alterações do pensamento e da percepção são decorrentes do mau contato com a realidade (Villemor-Amaral et al., 2005).

Há perturbações de funções básicas relacionadas ao senso de individualidade, unicidade e de direção de si mesmo. Os pacientes relatam que seus pensamentos, sentimentos e atos são conhecidos ou partilhados por outros sem sua vontade ou intenção. Esses, geralmente são explicados pelo paciente, como sendo eventos sobrenaturais ou fantásticos. Alucinações auditivas são mais frequentemente relatadas e o paciente se queixa de vozes que comentam sobre seus comportamentos ou pensamentos (OMS, 1993).

Em relação à capacidade intelectual e cognitiva, Zimmer et al. (2008) apontam para algumas discordâncias na literatura sobre a avaliação qualitativa e quantitativa de déficits cognitivos específicos nessa área. Segundo os autores, diversos instrumentos são utilizados sem o cuidado necessário no que diz respeito à validade e confiabilidade. Estima-se que $40 \%$ a $60 \%$ dos pacientes esquizofrênicos têm algum déficit cognitivo (Adad, Castro, \& Mattos, 2000). Segundo estes autores, estudos salientam que a atenção, a memória e as funções executivas são os principais processos cognitivos afetados. Dessa forma, sugerem que alterações e rebaixamento intelectual, prejuízos na linguagem e outros aspectos ligados à inteligência seriam secundários às alterações da atenção, memória e funções executivas (Pegoraro, Ceará, \& Fuentes, 2014). 
Neste caso, o último grupo de autores ainda sugere que pacientes com esquizofrenia apresentam lentificação para desempenhar tarefas atencionais, 0 que acaba prejudicando seu desempenho. Em relação à memória, eles apresentam dificuldades na organização dos processos de armazenamento e recuperação das informações, não se beneficiando de pistas fonéticas ou semânticas. No que diz respeito às funções executivas, esses indivíduos, apresentam prejuízos nas habilidades de abstração e flexibilização na resolução de problemas (Pegoraro et al., 2014).

O diagnóstico da esquizofrenia até os dias atuais não é algo simples, principalmente por não haver um sintoma patognomônico ou curso uniforme para todos os pacientes (Valença \& Nardi, 2015). Os sintomas da esquizofrenia podem ser classificados em três grandes grupos: 1) positivos, 2) negativos e 3 ) desorganizados/defeituosos. O primeiro grupo corresponde às características que estão ausentes em não-pacientes e presentes em pacientes esquizofrênicos, são exemplos as alucinações e delírios (Cheniaux, 2015; Johann \& Vaz, 2006a; Londere, Massuda, \& Gama, 2015).

Os sintomas negativos correspondem aos comportamentos que estão presentes nos pacientes e ausentes nos não-pacientes como, por exemplo, o embotamento afetivo, alogia (falta de lógica, discurso ou pensamento incompreensível), avolição (falta de vontade para realizar qualquer tipo de atividade) e anedonia (incapacidade de sentir satisfação/prazer em tarefas). Por fim, os sintomas desorganizados/defeituosos são características presentes tanto nos não-pacientes quanto nos pacientes, porém, nesses últimos, aparecem de forma caótica como comportamentos bizarros, afeto inapropriado e desordem do pensamento (Gama, Souza, Lobato, \& Belmonte-de-Abreu, 2004; Johann \& Vaz, 2006a).

Segundo os critérios de classificação da CID-10 (OMS, 1993), diagnosticase com esquizofrenia o indivíduo que apresentar ao menos um dos seguintes sinais ou sintomas: a) Eco de pensamento, inserção ou bloqueio de pensamento ou irradiação de pensamento, b) Delírios de controle, influência ou passividade, claramente relacionados a movimentos do corpo ou membros ou pensamentos, ações ou sensações específicos; percepção delirante, c) Vozes alucinatórias fazendo um comentário contínuo sobre o comportamento do paciente ou discutindo entre si ou outros tipos de vozes alucinatórias vindas de alguma parte do corpo, d) Delírios persistentes de outros tipos, culturalmente inapropriados e completamente impossíveis, tais como identidade religiosa ou política, poderes e habilidades sobre-humanas (ex., ser capaz de controlar o tempo ou entrar em comunicação com seres alienígenas).

Ou ainda pelo menos dois dos seguintes sinais ou sintomas: e) Alucinações persistentes em qualquer modalidade, que ocorram cotidianamente durante pelo menos um mês, quando acompanhadas por delírios sem conteúdos afetivos claros ou quando acompanhadas por ideias supervalorizadas persistentes, f) 
Neologismos, quebras ou interpolação no curso do pensamento, resultando em incoerência ou fala irrelevante, g) Comportamento catatônico, tal como excitação, postura inadequada ou flexibilidade, negativismo, mutismo e estupor, h) Sintomas negativos como apatia marcante, escassez de fala e embotamento ou incongruência de respostas emocionais (OMS, 1993).

A esquizofrenia pode ser subdivida em um espectro contendo nove subtipos de acordo com características peculiares de cada um. Também pode ser classificado de acordo com o padrão de curso (contínuo, episódico com déficit progressivo, episódico com déficit estável, episódico remitente, remissão incompleta, remissão completa, outro e período de observação menor do que um ano). Para o diagnóstico diferencial, o paciente deve atender aos critérios mínimos de classificação como esquizofrenia para, somente então, indicar um subtipo (OMS, 1993; Ortiz \& Lacaz, 2012).

Em relação aos subtipos têm-se as seguintes nomenclaturas para esquizofrenia: paranoide, hebefrênica, catatônica, indiferenciada, depressão pósesquizofrênica, residual, simples, outra esquizofrenia e não especificada. A esquizofrenia do tipo paranoide é marcada pela presença de delírios de perseguição, vozes alucinatórias que ameaçam o paciente ou lhe dão ordens, alucinações olfativas ou gustativas, além de sensações sexuais ou corporais. 0 começo tende a ser mais tardio quando comparado às formas hebefrênica e catatônica (OMS, 1993).

A esquizofrenia hebefrênica é caracterizada por mudanças afetivas proeminentes, comportamentos irresponsáveis e imprevisíveis. O afeto é superficial e acompanhado por risadas ou sorrisos, caretas, maneirismos e brincadeiras voltadas para si, sem contexto pertinente. Usualmente os primeiros sintomas são manifestados por volta dos 15 aos 25 anos. O tipo catatônico é marcado por perturbações psicomotoras que podem se alterar entre extremos como hipercinesia e estupor ou rigidez e negativismo. Aliado a isso, é comum o paciente hebefrênico manter uma mesma postura inapropriada ou bizarra por longo período de tempo (OMS, 1993).

O subtipo esquizofrenia indiferenciada é diagnosticado quando os comportamentos do paciente não se adequam a nenhum dos subtipos anteriormente relatados ou então não há predominância de comportamentos que justifiquem outro tipo de classificação. A depressão pós-esquizofrênica é caracterizada como um episódio depressivo prolongado que surgiu após a constatação da esquizofrenia. Nesse quadro, os sintomas padrões da esquizofrenia ainda estão presentes embora sua intensidade tenha diminuído consideravelmente (OMS, 1993). Nesse subtipo, é fundamental atentar-se para a presença de sinais ou sintomas da esquizofrenia anteriormente ao episódio depressivo.

Diagnostica-se esquizofrenia residual em casos que houve progressão clara de um estágio inicial para um estágio mais tardio onde os sintomas 
negativos são predominantes. A esquizofrenia simples é caracterizada pelo empobrecimento social, embotamento afetivo, inatividade e falta de objetivos para vida. Nesse subtipo os delírios e alucinações são ausentes ou não evidentes. Os outros dois subtipos "outra esquizofrenia" e "não especificada" são utilizados quando não há comportamentos claros que definam alguma das categorias previamente apresentadas (OMS, 1993).

Há diferenças entre os critérios e classificações utilizadas pela CID-10 e DSM-5. Em relação aos subtipos de esquizofrenia, a nova versão do DSM abandonou tais divisões por compreender que há pouca validade e as diferenças quanto ao curso da doença ou resposta ao tratamento não são alteradas (Araújo \& Lotufo Neto, 2014).

O tratamento de pacientes esquizofrênicos é realizado por meio da medicação antipsicótica e pode ser associado a tratamentos psicoterápicos, terapias ocupacionais, entre outros. Embora a farmacoterapia não seja curativa (Salim, 1987, Stroup, Marder, \& Lieberman, 2012), ela é considerada o tratamento primário em todos os tipos e estágios da esquizofrenia, contribuindo na redução do tempo de hospitalização (Gama, Souza, Lobato \& Belmonte-deAbreu, 2004). Têm-se, atualmente, dois principais tipos de antipsicóticos que atuam seletivamente no Sistema Nervoso Central (SNC): os de primeira e segunda geração (Johann \& Vaz, 2006a). Como principais representantes desses dois grupos de medicamentos, pode-se citar, respectivamente, o haloperidol e a clozapina. A escolha do tipo de droga deve levar em consideração os efeitos adversos e a eficácia em cada paciente.

Considerado uma droga de alta potência por atingir os mesmos resultados que outros medicamentos com uma dose até 20 vezes menor, o haloperidol é um derivado butirofenônico (Salim, 1987) que funciona bloqueando os receptores dopaminérgicos e diminuindo os sintomas positivos. Entretanto, essa medicação apresenta efeitos colaterais autonômicos e extrapiramidais importantes tais como sedação, hipotensão postural, parkinsonismo medicamentoso, síndrome neuroléptica maligna, entre outros (Johann \& Vaz, 2006b).

A clozapina, por sua vez, é considerada a primeira medicação antipsicótica com efeitos positivos quantitativos e qualitativos em relação aos antipsicóticos de primeira geração. Ela atua como antagonista em receptores 5-HT2A, D1, D4, alfa adrenérgico, colinérgico e histamínicos (Gama et al., 2004). Essa droga atua não apenas na redução dos sintomas positivos como também nos negativos, sendo eficiente nos aspectos psicossociais e na prevenção de comportamentos suicidas (Gama et al., 2004; Johann \& Vaz, 2006b). Embora os efeitos extrapiramidais sejam menos frequentes, a clozapina apresenta efeitos adversos tais como taquicardia, hipotensão, hipersalivação, sedação e convulsões. Segundo o estudo realizado por Gama et al. (2004) com 56 pacientes com esquizofrenia medicados com clozapina, os pacientes diagnosticados com esquizofrenia tipo paranoide 
apresentaram melhores resultados ao tratamento após seis meses de uso quando comparado aos demais tipos de esquizofrenia.

\section{Avaliação psicológica e os testes projetivos/expressivos}

As funções cognitivas são avaliadas por meio de instrumentos neuropsicológicos como inventários, escalas e testes de desempenho/capacidade. A partir dos testes, têm-se amostras de comportamentos que descrevem ou mensuram habilidades e características como emoção, inteligência, atenção, memória, percepção, entre outros (Abreu, Rivero, Coutinho, \& Bueno, 2014; Camargo, Bolognani, \& Zuccolo, 2014; Keefe \& Eesley, 2012; Malloy-Diniz, Paula, \& Sedó, 2014; Urbina, 2007). A partir desses dados, traça-se um perfil cognitivo e afetivo do paciente no qual se conhece as potencialidades e dificuldades dele. Determinados perfis são característicos de algumas psicopatologias, como é o caso da esquizofrenia.

A maioria dos testes que são utilizados em avaliações no contexto de saúde mental é classificada como de desempenho ou capacidade. São tarefas objetivas que mensuram conhecimentos, habilidades ou funções cognitivas específicas. As respostas do avaliando são consideradas como certas ou erradas e seu desempenho final é comparado com uma população específica de acordo com a faixa etária ou grau de escolaridade, entre outros (Urbina, 2007).

Quando se avalia a personalidade, que é um fator importantíssimo para se diagnosticar uma psicopatologia (Vieira, Fay, \& Neiva-Silva, 2007), são mais usualmente utilizados inventários, questionários e escalas. Nesses instrumentos o respondente deve assinalar afirmativas que ele julga serem condizentes com sua forma de agir e pensar. Não existem respostas certas ou erradas (Urbina, 2007).

O maior empecilho desse tipo de instrumento é que em psicopatologias graves, como a esquizofrenia, sabe-se que o paciente apresenta distorções - de moderada à severa - da percepção da realidade e da percepção de si mesmo (Vieira et al., 2007). Dessa forma, compreende-se que o uso de inventários não é o melhor recurso para esse tipo de avaliação com pacientes psiquiátricos cuja principal suspeita seja a alteração da capacidade de dizer sobre si da mesma forma como as outras pessoas o percebem. Os instrumentos projetivos/expressivos se apresentam como uma alternativa interessante que minimiza a manipulação das respostas uma vez que são compostos por características ambíguas e abstratas (Anastasi \& Urbina, 2000; Villemor-Amaral et al., 2005; Urbina, 2007).

Outra vantagem do uso dos instrumentos projetivos é que muitos deles são classificados como não-verbais, ou seja, o respondente emite pouca ou nenhuma resposta verbal. Essa característica é interessante quando o paciente apresenta algum comprometimento na expressão ou comunicação verbal. Atualmente há um movimento entre os estudiosos da avaliação psicológica no 
sentido de revisar a nomenclatura utilizada por essa técnica de investigação. Miguel (2014) esclarece que o uso do termo "projetivo" pode causar a errônea impressão de que esse instrumento é de uso exclusivo da psicanálise devido ao mecanismo de defesa denominado por Freud (1913/2006) de projeção. O autor indica que a substituição do termo por outros como "expressivo", "de desempenho", "implícito" ou "de resposta livre", evitaria tal mal-entendido, uma vez que o fenômeno projeção não está, necessariamente, vinculado à teoria psicanalítica.

As técnicas projetivas/expressivas têm como ponto em comum o material que, ao mesmo tempo é concreto, ambíguo e vago (Anastasi \& Urbina, 2000; Chabert, 2004). As instruções geralmente são breves e gerais e por serem tarefas minimamente estruturadas, permitem ao avaliando ampla possibilidade de respostas (Anastasi \& Urbina, 2000).

Os principais testes projetivos usados para avaliação no contexto da saúde mental, segundo Vieira et al. (2007) são o Teste de Zulliger (Zulliger), o Teste de Apercepção Temática (TAT) e HTP (Teste House-Tree-Person). Adicionalmente, verificou-se que outros instrumentos como o Teste das Pirâmides Coloridas de Pfister e o Teste das Manchas de Tinta de Rorschach têm ganhado espaço na avaliação em contexto da saúde mental (Lukasova, Zanin, Chucre, Macedo \& Macedo, 2010; Vieira \& Villemor-Amaral, 2015; Villemor-Amaral et al., 2005, entre outros).

O Zulliger (Villemor-Amaral \& Primi, 2012) e o Rorschach (Exner Jr. \& Sendín, 1999; Exner Jr., 1999; Vaz, 1997) consistem em cartões com manchas de tintas às quais é solicitado ao respondente que diga com o que acha que elas se parecem (etapa de associação). São apresentados todos os cartões (três cartões no Zulliger e dez no Rorschach) e, posteriormente, é solicitado ao respondente que diga onde, e quais aspectos da mancha lhe deram a impressão de cada um das respostas apresentadas (etapa de inquérito).

Atualmente o sistema para codificação e interpretação dos dados do Zulliger é o Sistema Compreensivo para administração individual (VillemorAmaral \& Primi, 2012). Em relação ao Rorschach, há quatro possibilidades de sistemas aprovados pelo Conselho Federal de Psicologia (CFP) para uso, são eles: Sistema Compreensivo (Exner Jr. \& Sendín, 1999; Exner, Jr., 1999), Sistema de Classificação de Klopfer (Vaz, 1997) e Escola Francesa (Rausch de Traubenberg, 1975; Pasian, 2000) e Rorschach Clínico ou Sistema Aníbal Silveira (Pasian \& Loureio, 2010). Pesquisas estão sendo desenvolvidas para validação do sistema Rorschach Perfomance Assessment System (R-PAS) (Meyer, Viglione, Mihura, Erard, \& Erdberg, 2011), também para uso no Rorschach.

O TAT (Murray, 2005) é composto por 31 pranchas com impressos de imagens de situações cotidianas e ambíguas e uma prancha totalmente branca. O respondente deve contar uma história a partir do estímulo de cada das 20 
pranchas pré-selecionadas pelo aplicador de acordo com o sexo e faixa etária (Vieira et al., 2007).

O HTP (Tardivo, 2003) consiste em uma técnica gráfica onde se solicita que o avaliando desenhe em tons acromáticos ou cromáticos uma sequência de elementos. Após isso, o sujeito é instruído a dizer sobre cada desenho, podendo ser utilizado um roteiro de perguntas gerais e específicas para cada desenho (Vieira et al., 2007). O Pfister (Villemor-Amaral, 2012) consiste em um esquema de pirâmides que devem ser preenchidas com quadrículos coloridos (VillemorAmaral et al., 2005).

O PMK (Galland de Mira, 2002), é um instrumento em que o sujeito deve fazer diferentes tipos de traçados feitos em seis páginas distintas (Vasconcelos, Sampaio \& Do Nascimento, 2013). Por fim, o Palográfico (Alves \& Esteves, 2004) avalia os vários "palos" (traços verticais) que o respondente deve fazer em um período de tempo determinado. Embora ambos os instrumentos não sejam comumente utilizados na avaliação no contexto de saúde mental, esses foram incluídos na presente pesquisa atendendo aos critérios de seleção de instrumentos, conforme será discutido em Método.

De forma geral, todos esses instrumentos avaliam além de traços e características da personalidade, o desempenho cognitivo do avaliando uma vez que é necessária certa habilidade intelectual e cognitiva para realizar a tarefa (Miguel, 2014). De forma específica, os testes projetivos avaliam a capacidade do respondente de seguir tarefas, e expressar, por meio dos estímulos particulares de cada instrumento, a forma como percebe emocionalmente e cognitivamente o mundo.

Tendo em vista a necessidade de instrumentos que forneçam indicadores sensíveis para psicopatologias, devido principalmente à dificuldade do diagnóstico e alteração na percepção da realidade (que compromete os resultados de alguns instrumentos, como o caso de inventários de auto relato), buscou-se, no presente estudo, investigar o que se têm produzido sobre a temática do uso de instrumentos projetivos/expressivos na avaliação psicológica de pacientes com esquizofrenia no Brasil. A partir dessa busca, objetivou-se apresentar e discutir as pesquisas encontradas a fim de se verificar as contribuições e possíveis limites do estudo ou da técnica.

\section{MÉTODO}

Buscaram-se trabalhos publicados nas bases de dados eletrônicos Scielo, Lilacs, Medline, Pepsic e Index Psicologia. Foram usados conjuntos de descritores contendo, cada conjunto, duas palavras-chave fixas e um descritor que correspondia aos nomes usuais de alguns instrumentos projetivos. Dessa forma, usaram-se sempre duas palavras-chave: avaliação e esquizofrenia e a terceira 
palavra variou de acordo com o teste: HTP, Pfister, Palográfico, PMK, Rorschach, TAT, Zulliger.

A escolha dos instrumentos projetivos/expressivos foi baseada na lista de testes com parecer favorável disponíveis no SATEPSI até agosto de 2015. Optouse por não usar o descritor projetivo, pois há, atualmente, variadas nomenclaturas em sua para se referir a essa técnica (Miguel, 2014), o que poderia prejudicar a busca caso se optasse pelo uso da "classificação" do instrumento em detrimento do nome dos instrumentos em si. Foram selecionados apenas instrumentos cuja idade de aplicação fosse específica para adultos.

A escolha dessas cinco bases de dados teve como objetivo alcançar o maior número de produções científicas brasileiras nesse tema. Como critério de inclusão, foram selecionados apenas artigos publicados em periódicos indexados, dissertações e teses brasileiras, que pudessem ter o seu texto completo acessado gratuitamente. Como critério de exclusão, foram eliminados livros e capítulos de livros por se considerar que esses muitas vezes não passaram por avaliações mais rigorosas por pares. Não houve critério para seleção do ano de publicação dos trabalhos.

\section{RESULTADOS}

A busca resultou em dez artigos, apresentados na Tabela 1 conforme o ano de publicação em ordem crescente. Os artigos foram lidos na íntegra e separados conforme instrumento projetivo/expressivo utilizado.

Tabela 1. Relação de artigos selecionados a partir dos critérios estabelecidos

\begin{tabular}{|c|c|c|c|}
\hline Título & Ano & Autor(es) & Técnica \\
\hline $\begin{array}{l}\text { Caracterização da percepção do real, de } \\
\text { pacientes esquizofrênicos, avaliados } \\
\text { através das técnicas das pirâmides } \\
\text { coloridas de Pfister }\end{array}$ & 1993 & Carnio \& Loureiro & Pfister \\
\hline $\begin{array}{l}\text { A representação de si na esquizofrenia } \\
\text { através do psicodiagnóstico de } \\
\text { Rorschach }\end{array}$ & 2000 & Santos & Rorschach \\
\hline $\begin{array}{l}\text { O teste de Pfister e sua contribuição } \\
\text { para o diagnóstico da esquizofrenia } \\
\text { Condições afetivas e de relacionamento } \\
\text { interpessoal em homens portadores de } \\
\text { esquizofrenia em tratamento com } \\
\text { haloperidol ou clozapina }\end{array}$ & 2005 & $\begin{array}{l}\text { Villemor-Amaral } \\
\text { et al. } \\
\text { Johann \& Vaz }\end{array}$ & Rorschach \\
\hline $\begin{array}{l}\text { Avaliação de aspectos cognitivos em } \\
\text { homens portadores de esquizofrenia } \\
\text { em tratamento com haloperidol ou }\end{array}$ & 2006 & Johann \& Vaz & Rorschach \\
\hline
\end{tabular}


clozapina

\begin{tabular}{|c|c|c|c|}
\hline $\begin{array}{c}\text { Representação de objeto e organização } \\
\text { psíquica: Integração dinâmica dos } \\
\text { dados do Rorschach }\end{array}$ & 2006 & Santos \& Vaz & Rorschach \\
\hline $\begin{array}{l}\text { Subtipos de esquizofrenia e indicadores } \\
\text { do psicodiagnóstico de Rorschach }\end{array}$ & 2008 & Bereta & Rorschach \\
\hline $\begin{array}{c}\text { Análise dos movimentos oculares de } \\
\text { pacientes com esquizofrenia durante a } \\
\text { exploração visual de figuras de testes } \\
\text { projetivos }\end{array}$ & 2010 & Lukasova et al. & $\begin{array}{l}\text { Rorschach } \\
\text { \& TAT }\end{array}$ \\
\hline $\begin{array}{c}\text { A técnica de Rorschach e os critérios da } \\
\text { CID-10 para o diagnóstico da } \\
\text { esquizofrenia }\end{array}$ & 2012 & $\begin{array}{l}\text { Resende \& } \\
\text { Argimon }\end{array}$ & Rorschach \\
\hline $\begin{array}{l}\text { Evidências de validade do Rorschach } \\
\text { Performance Assessment System no } \\
\text { diagnóstico da esquizofrenia }\end{array}$ & 2015 & $\begin{array}{l}\text { Vieira \& Villemor- } \\
\text { Amaral }\end{array}$ & Rorschach \\
\hline
\end{tabular}

Dentre os artigos localizados, sete utilizavam exclusivamente o Rorschach como instrumento projetivo de avaliação, dois empregavam o Pfister e um o TAT em conjunto com o Rorschach. Oito artigos (80\%) foram publicados nos últimos dez anos.

O estudo de Carnio e Loureiro (1993) objetivou fazer um levantamento dos índices característicos da percepção e adaptação à realidade em pacientes esquizofrênicos. Para isso, as pesquisadoras aplicaram o teste das Pirâmides Coloridas de Pfister em 20 pacientes que estavam internados no setor psiquiátrico e tinham diagnóstico de esquizofrenia. A partir da análise dos índices de organização lógica e síndromes adaptativas verificou-se que essa amostra apresentava um acentuado prejuízo na adaptação e percepção da realidade, demonstrando certo estreitamento e afastamento no contato com a realidade.

Mais de uma década depois, Villemor-Amaral et al. (2005) desenvolveram um estudo semelhante ao de Carnio e Loureiro (1993). Os autores investigaram quais indicadores do Pfister apareceriam com maior frequência em um grupo de pacientes esquizofrênicos quando comparados a um grupo de não-pacientes. Participaram 20 sujeitos diagnosticados com esquizofrenia e 110 indivíduos sem qualquer diagnóstico psiquiátrico prévio.

Os autores analisaram a frequência de cores e os aspectos formais das pirâmides por meio de análise de regressão logística. Observaram que as cores vermelha e marrom eram mais frequentemente utilizadas nas três pirâmides executadas pelo grupo de pacientes quando comparado ao grupo controle. Aliado a isso, constataram incidência de tapetes furados e desequilibrados significativamente maior nos pacientes enquanto que foi observada uma menor ocorrência de formações estratificadas no mesmo grupo. 
A partir de análise estatística, verificaram que é possível diagnosticar corretamente $70 \%$ dos esquizofrênicos com base nesses indicadores, havendo, entretanto, um risco de $43 \%$ de falsos positivos. As cores vermelha e marrom estão associadas à impulsividade e incontinência de reações, indicando dificuldades de adaptação à realidade enquanto os tapetes furados e desiquilibrados estão associados a um pensamento cognitivamente mais simplista, menos elaborado.

Comparando ambos os estudos encontrados, verificaram-se algumas discordâncias de resultados. Enquanto no estudo de Carnio e Loureiro (1993) foi encontrado uma diminuição da cor branca em pirâmides executadas por pacientes esquizofrênicos, no estudo de Villemor-Amaral et al. (2005) não foram encontrados indicadores nem de diminuição nem de aumento da mesma cor. Aliado a isso, não foi encontrado, no estudo de Villemor-Amaral et al. (2005) diferença significativa no uso dessa cor entre pacientes e não-pacientes.

Outra discordância está relacionada à ocorrência de síndromes de adaptação ao ambiente (SAA). No estudo de Carnio e Loureiro (1993) foi encontrada uma grande incidência de combinações típicas de afastamento, (cerca de 45\%) enquanto que na pesquisa de Villemor-Amaral et al. (2005) essa porcentagem caiu para $25 \%$. Tais discrepâncias podem ser explicadas pela reduzida amostra de pacientes coletada em ambos os estudos.

Apesar dos indicadores na produção das pirâmides - que são mais frequentemente apresentados na população esquizofrênica - serem divergentes o último grupo de autores evidenciaram que há discrepâncias na execução do teste de Pfister em esquizofrênicos quando comparados à população geral, sem diagnóstico psiquiátrico.

No estudo de Villemor-Amaral et al. (2005) foi feito um cálculo probabilístico de diagnóstico de esquizofrenia a partir dos resultados obtidos pelo Pfister. Os autores verificaram, porém, que a porcentagem de falsos positivos é relativamente alta. Desta forma, como apontado pelos autores, uma avaliação, seja ela neuropsicológica ou psicológica vai além da aplicação de testes e instrumentos de mensuração (Primi, 2010). Outros indicadores, como a entrevista com o sujeito e informantes, a observação, entre outros; devem ser utilizados (Urbina, 2007). Assim, embora seja relevante a verificação de possibilidades de predição de um transtorno em testes como o Pfister, a mesma avaliação estatística de outros instrumentos deveria ser realizada, possibilitando ao psicólogo, em sua prática, reunir instrumentos mais refinados a partir de uma hipótese diagnóstica.

O estudo de Santos (2000) avaliou 20 pacientes esquizofrênicos por meio do teste de Rorschach, segundo a interpretação da escola francesa. A partir da análise dos dados, o autor observou que os pacientes apresentavam indicadores de severo comprometimento no que diz respeito à capacidade de representação e organização do eu. Em relação à percepção de si, de seu mundo interno, o 
autor verificou que os pacientes com esquizofrenia se apresentavam precariamente ordenados e integrados, apresentando pouca diferenciação entre o eu e o objeto externo. Essa indiferenciação, segundo o autor, levaria a uma cisão severa das funções psíquicas (percepção, cognição e afetividade).

Uma revisão dos principais modelos de representações temáticas e conteúdos presentes nas verbalizações feitas por pacientes no Rorschach foi realizada por Santos e Vaz (2006). Os autores verificaram quais eram as principais abordagens utilizadas até o momento e quais modelos teóricos eram utilizados para conceituar a representação de si. Os autores constataram que desde sua criação, novas abordagens na avaliação e na organização das temáticas desenvolvidas no Rorschach foram incorporadas ao esquema clássico de aferição das verbalizações e respostas. Esses desenvolvimentos conceituais, segundo os autores, refletem as teorias cognitivas/desenvolvimentistas e psicanalíticas. Assim, o papel das relações de objeto no desenvolvimento da capacidade representacional é verificado tanto a partir dos aspectos cognitivos/estruturais quanto do afetivo/temático, dependendo do referencial adotado.

Johann e Vaz (2006a) compararam os dados de protocolos de Rorschach de um grupo de pacientes com esquizofrenia medicados com haloperidol $(n=14)$ e outro com clozapina $(n=16)$ por meio do Sistema de Classificação de Klopfer. Os autores verificaram que os pacientes medicados com clozapina apresentaram indicativos de controle geral sobre seus impulsos, capacidade de reparação, capacidades afetivas e emocionais para estabelecer relacionamentos interpessoais. Os pacientes que faziam uso de haloperidol apresentaram apenas indicativos de capacidade de controle sobre impulsos. Dessa forma, os autores concluem que o uso de clozapina pode favorecer a redução de sintomas da esquizofrenia referentes à interação interpessoal.

Usando a mesma amostra clínica, Johann e Vaz (2006b) avaliaram os aspectos cognitivos de ambos os grupos de pacientes esquizofrênicos. Dentre os indicadores do Rorschach, os autores escolheram como parâmetro para essa comparação, o número de respostas produzidas, a localização e a incidência e qualidade de forma pura e movimento animal. Assim como no estudo anterior, os autores verificaram que há um melhor desempenho entre os pacientes usuários de medicação de segunda geração. Desta forma, o grupo de pacientes medicados com clozapina apresentavam mais indicativos de capacidade de produção e adaptação à tarefa e raciocínio lógico mais desenvolvido do que o outro grupo. A partir desses resultados os autores inferem que o uso de clozapina ajuda na organização do raciocínio lógico de pacientes diagnosticados com esquizofrenia.

O estudo de Bereta (2008) analisou, baseando-se na classificação francesa, quatro protocolos de Rorschach de pacientes com diagnóstico de esquizofrenia, cada um com um subtipo diferente (hebefrênico, simples, residual 
e paranoide). Seu objetivo era verificar se haveriam indicadores no Rorschach que caracterizassem cada subtipo de esquizofrenia. A autora verificou que não há indicadores, dentro da nomenclatura francesa, que caracterizam especificamente os subtipos do transtorno esquizofrênico.

Lukasova et al. (2010) investigaram o padrão de movimento ocular em 10 pacientes psiquiátricos e 10 não-pacientes durante a apresentação das pranchas do Rorschach e os cartões do TAT. Foi observado que os pacientes com esquizofrenia exibiram um padrão do movimento ocular diferente do grupo controle. Os primeiros apresentaram menos fixações nas pranchas do Rorschach e nos cartões do TAT com faces humanas. Dessa forma os autores concluem que há alterações na exploração visual em pacientes esquizofrênicos e que tal alteração pode ser relacionada à capacidade de integração da informação visual abstrata.

Resende e Argimon (2012) correlacionaram as variáveis da técnica de Rorschach e os oito grupos de sinais e sintomas da CID-10 para a esquizofrenia. As autoras se basearam no Sistema de Classificação de Klopfer e em algumas categorias de codificação do Sistema Compreensivo para analisar 80 protocolos de pacientes diagnosticados com esquizofrenia. Contatou-se que há correlações entre os indicadores do Rorschach e os grupos de sintomas da CID-10 embora se tratem de instrumentos com referenciais diferentes no campo da psicopatologia.

Por fim, mais recentemente foi publicado o trabalho de Vieira e VillemorAmaral (2015) que buscou evidências de validade para o Rorschach pelo sistema R-PAS para o contexto brasileiro em pacientes com esquizofrenia. Para tanto, foram analisados 35 protocolos de pacientes diagnosticados com a enfermidade e 35 protocolos de não pacientes. Os resultados obtidos evidenciam diferenças estatisticamente significativas para variáveis como qualidade formal, códigos cognitivos, movimento animal, entre outros. Esses resultados sugerem que o sistema R-PAS é válido para o contexto brasileiro.

Dos dez estudos apresentados, oito deles foram desenvolvidos utilizando o Rorschach como instrumento em comum no diagnóstico da esquizofrenia. Como pôde ser observado, foram usados quatro principais sistemas de codificação e interpretação. Santos (2000) e Bereta (2008) basearam-se nas interpretações da escola francesa, Johann e Vaz (2006a), Johann e Vaz (2006b) e Resende e Argimon (2012) utilizaram os Sistema de Classificação de Klopfer, sendo que as últimas autoras o associaram ao Sistema Compreensivo. Por fim, Vieira e Villemor-Amaral utilizaram o Sistema R-PAS em seu estudo. O estudo de Lukasova et al. (2010) não objetivou codificar as repostas do Rorschach ou do TAT, mas sim apresentá-las como estímulos. Assim, não houve opção por sistema de interpretação. 


\section{DISCUSSÃO}

Todos os autores que utilizaram o Rorschach como instrumento de avaliação de características da esquizofrenia obtiveram resultados promissores no que diz respeito à possibilidade de uso desse teste na avaliação psicológica desse transtorno. Esse instrumento demonstrou, em todos os sistemas de classificação analisados pelos autores, evidências de validade no que diz respeito à identificação de indicadores psicopatógicos da esquizofrenia.

Com exceção dos dois últimos estudos, verificou-se que a amostra utilizada nas pesquisas é reduzida, o que dificulta a generalização dos dados. Sabe-se que a técnica de Rorschach, independentemente do sistema utilizado para codificação das respostas é bastante complexa e demorada, o que dificulta a ampliação do número da amostra. Aliado a isso, a população critério utilizada nesses estudos também não é facilmente localizada dado a sua baixa incidência de casos onde não há outros transtornos associados, lesões cerebrais ou antecedentes de abuso de drogas ou álcool.

Dentre os sete instrumentos projetivos procurados nas bases de dados utilizadas na presente pesquisa, apenas três deles foram utilizados como instrumentos de pesquisa para avaliação psicológica de pacientes com esquizofrenia no Brasil. Aventou-se algumas hipóteses para tal constatação.

$\mathrm{O}$ teste PMK, apenas recentemente reinserido na lista de testes com parecer favorável pelo CFP, é mais usualmente utilizado em contexto de avaliação de motoristas (Rueda \& Lamounier, 2006) e desempenho no trabalho (Vasconcelos, Sampaio, \& Do Nascimento, 2013).

Embora seja um teste que se classifique como capaz de avaliar a personalidade de sujeitos, suas pesquisas e uso não são voltados para o contexto psiquiátrico (Vasconcelos, Do Nascimento, \& Sampaio, 2011). Aliado a isso, em alguns estudos como o de Sisto (2010) não foram encontradas evidências de interpretabilidade para quase nenhum dos seis fatores propostos pelo instrumento.

Esse dado deve ser considerado, pois há indicadores na literatura de que o instrumento não é capaz de avaliar aquilo que se propõe apesar de ter sido aprovado pelo CFP a partir da apresentação de novos estudos de validade. Ressalta-se, a partir dessa observação, que embora haja medidas que estabeleçam critérios mínimos de validade, precisão e padronização para que um instrumento seja aprovado pelo CFP (2003), de acordo com a resolução 2/2003, é imprescindível que o psicólogo esteja apto a verificar, junto ao manual do teste, sua qualidade psicométrica.

Das pesquisas encontradas com o Palográfico, algumas são voltadas para o contexto de seleção profissional (Godoy \& Noronha, 2005), impulsividade e compulsão alimentar (Pereira \& Chehter, 2011) e análise do perfil psicológico de 
profissionais que atuam na área da saúde (Senden, Lopes, Lopes do Nascimento, Terroso \& Argimon, 2013).

Embora os instrumentos HTP e Zulliger apresentem indicadores psicopatológicos em suas interpretações e classificações, também não foram localizados estudos dentro do recorte estabelecido sobre o uso na avaliação da esquizofrenia. Apesar disso, foram encontrados estudos que avaliam por meio de ambos ou um dos instrumentos, indicadores psicopatológicos como depressão e transtornos dissociativos e transtornos de personalidade histriônicos (Pereira, 2011; Villemor-Amaral \& Machado, 2011).

Em relação ao TAT foi encontrado um segundo estudo sobre avaliação da população diagnosticada com esquizofrenia (Henriques-Calado \& Duarte-Silva, 2011). Esse estudo, porém, foi excluído na seleção, atendendo aos critérios de exclusão. Isso se deveu ao fato do estudo ter sido realizado em uma amostra não-brasileira.

Compreende-se que, embora o número de estudos encontrados tenha sido relativamente baixo, as contribuições dos instrumentos projetivos para a avaliação neuropsicológica de transtornos psicopatológicos como é o caso da esquizofrenia é fundamental. Esses instrumentos, como já foi destacado, são menos diretivos e requerem poucas instruções, o que facilita seu uso em diversos contextos (Villemor-Amaral et al., 2005).

Alguns instrumentos, como é o caso do Rorschach e TAT, exigem refinado conhecimento do aplicador e seu processo de codificação e análise é mais complexo quando comparado aos demais instrumentos projetivos (Miguel, 2014). A despeito disso, observou-se que, dentre os instrumentos projetivos utilizados na avaliação e estudo neuropsicológico da esquizofrenia, o Rorschach é o teste mais frequentemente utilizado em tais pesquisas.

Uma hipótese para tal ocorrência se dá pela riqueza de dados possíveis de serem verificados por meio da técnica de Rorschach, em todos os sistemas de codificação e interpretação. Esse instrumento possibilita a avaliação não apenas dos traços de personalidade e conduta dos respondentes, mas também a verificação de aspectos cognitivos como a percepção da realidade e a verificação de aspectos do relacionamento interpessoal como a necessidade de contato interpessoal (Meyer et al., 2011). Assim, embora o teste de Rorschach seja um instrumento de complexa aplicação, codificação e interpretação, ele se apresenta como um recurso com ampla possibilidade de uso.

Fora do Brasil a literatura sobre instrumentos projetivos como o Rorschach na avaliação ou mensuração de indicadores em pacientes com esquizofrenia é vasta. Estuda-se a evidência de indicadores de desordem do pensamento em parentes próximos de pacientes já diagnosticados (Gooding et al., 2012), relação entre desistência de tratamento e relacionamento interpessoal (Bouvet \& Cleach, 2011) e a avaliação das habilidades de socialização dessa população (Moore, Viglione, Rosenfarb, Patterson, \& Mausbach, 2013), entre outros estudos (Kimhy 
et al., 2007; Lukasova et al., 2010; Roisko, Wahlberg, Hakko, Wynne, \& Tienari, 2011; Rosenbaum, Andersen, Knudsen, \& Lorentzen, 2012; DzamonjaIgnjatovic, Smith, Jocic, \& Milanovic, 2013)

O objetivo desse estudo foi verificar o que se tem produzido na literatura brasileira sobre avaliação psicológica de pacientes diagnosticados com esquizofrenia por meio de instrumentos projetivos/expressivos. A maioria das pesquisas localizadas a partir dos critérios de inclusão e exclusão, utilizou a técnica das Manchas de Tinta de Rorschach como principal instrumento de investigação. Muitas das técnicas atualmente aprovadas pelo CFP não possuem pesquisas nessa área, embora se proponham a avaliar a personalidade e psicopatologias.

Verificou-se que fora do Brasil diversas pesquisas com a população esquizofrênica foram desenvolvidas. Isso contribui para melhor compreensão desse transtorno e melhor aprimoramento e desenvolvimento de instrumentos que sejam mais refinados quando se trata do diagnóstico de psicopatologias. Sendo assim, sugere-se que mais pesquisas sejam realizadas com a população psiquiátrica, enriquecendo não apenas o saber científico, mas possibilitando o desenvolvimento de novas ferramentas para diagnóstico e, possivelmente, intervenção.

\section{REFERÊNCIAS}

Abreu, N., Rivero, T. S., Coutinho, G, \& Bueno, O. F. A. (2014). Neuropsicologia da aprendizagem e memória. In D. Fuentes (Ed.), Neuropsicologia: Teoria e prática. (pp. 103-114). Porto Alegre: Artmed.

Adad, M. A., Castro, R., \& Mattos, P. (2000). Aspectos neuropsicológicos da esquizofrenia. Revista Brasileira de Psiquiatria, 22(1 Supl.), 31-34. doi: 10.1590/S1516-44462000000500011

Alves, I. C. B., \& Esteves, C. (2004). O teste palográfico na avaliação da personalidade. São Paulo: Vetor.

Anastasi, A., \& Urbina, S. (2000). Testagem psicológica. Porto Alegre, RS: Artes Médicas Sul.

Araújo, A. C. \& Lotufo Neto, F. (2014). A nova classificação americana para os transtornos mentais - O DMS-5. Revista Brasileira de Terapia Comportamental e Cognitiva, 16(1), 67-82.

Bereta, F. A. (2008). Subtipos de esquizofrenia e indicadores do psicodiagnóstico de Rorschach. (Monografia). Universidade de São Paulo, Ribeirão Preto.

Bouvet, C., \& Cleach, C. (2011). Les ruptures de prise en charge chez des patients d'un centre de soin de réadaptation psychiatrique et leus liens avec les capacites relationnelles, les relations d'objets intériorisées et l'intensité des troubles. L'Encéphale, 37, 19-26. doi: 10.1016/j.encep.2010.03.008 
Camargo, C. H. P., Bolognani, S. A. P., \& Zuccolo, P. F. (2014). O exame neuropsicológico e os diferentes contextos de aplicação. In D. Fuentes (Ed.), Neuropsicologia: Teoria e prática. (pp. 77-92). Porto Alegre, RS: Artmed.

Carnio, E. C. \& Loureiro, S. R. (1993). Caracterizações da percepção real, de pacientes esquizofrênicos, avaliados através das técnicas das Pirâmides Coloridas de Pfister. Psico, 24(1), 35-47.

Chabert, C. (2004). Psicanálise de métodos projetivos. São Paulo, SP: Vetor.

Cheniaux, E. (2015). Psicopatologia e diagnóstico da esquizofrenia. In A. E. Nardi, J. Quevedo, \& A. G. Silva (Eds.), Esquizofrenia: Teoria e clínica (pp. 37-44). Porto Alegre, RS: Artmed.

Conselho Federal de Psicologia. SATEPSI. Recuperado de: http://satepsi.cfp.org.br

Conselho Federal de Psicologia. (2003). Resolução $n^{\circ}$ 02/2003. Brasília: Conselho Federal de Psicologia. Recuperado de: http://site.cfp.org.br/resolucoes/resolucao-n-2-2003/

Dzamonja-Ignjatovic, T., Smith, B. L., Jocic, D. D., \& Milanovic, M. (2013). A comparison of new and revised Rorschach measures of schizophrenic functioning in a Serbian clinical sample. Journal of Personality Assessment, 95(5), 471-478. doi: 10.1080/00223891.2013.810153

Exner Jr., E. \& Sendín, C. (1999). Manual de interpretação do Rorschach para o sistema compreensivo. São Paulo, SP: Casa do Psicólogo.

Exner Jr. (1999). Manual de classificação do Rorschach para o sistema compreensivo. São Paulo, SP: Casa do Psicólogo.

Freud, S. (2006). Totem e tabu. In Obras psicológicas completas de Sigmund Freud: Edição standard brasileira (pp. 11-163). Vol. 13. Rio de Janeiro: Imago. (Original publicado em 1913).

Galland de Mira, A. M. (2002). PMK: Psicodiagnóstico Miocinético. São Paulo: Vetor.

Gama, C. S., Souza, C. M., Lobato, M. I., \& Belmonte-de-Abreu, P. S. (2004). Relato do uso de clozapina em 56 pacientes atendidos pelo programa de atenção à esquizofrenia refratária da secretaria da saúde e do meio ambiente do estado do Rio Grande do Sul. Revista Psiquiatria, 26(1), 21-28. doi: $10.1590 /$ S0101-81082004000100004

Godoy, S. L. \& Noronha, A. P. P. (2005). Instrumentos psicológicos utilizados em seleção profissional. Revista do Departamento de Psicologia, 17(1), 139159. doi: 10.1590/S0104-80232005000100011

Gooding, D. C., Coleman, M. J., Roberts, S. A., Shenton, M. E., Levy, D. L., \& Erlenmeyer-Kimling, L. (2012). Thought disorder in offspring of schizophrenic parentes: Findings from the New York High-Risk Project. Schizophrenia Bulletin, 38(2), 263-271. doi: 10.1093/schbul/sbq061 
Henriques-Calado, J., \& Duarte-Silva, M. E. (2011). A esquizofrenia e a demência no teste de apercepção temática: Uma análise da angústia e da relação de objeto. Contextos Clínicos, 4(2), 67-79. doi: 10.4013/ctc.2011.42.01

Johann, R. V. O., \& Vaz, C. E. (2006a). Avaliação de aspectos cognitivos em homens portadores de esquizofrenia em tratamento com haloperidol ou clozapina. Jornal Brasileiro de Psiquiatria, 55(3), 202-207. doi: 10.1590/S0047-20852006000300005

Johann, R. V. O., \& Vaz, C. E. (2006b). Condições afetivas e de relacionamento interpessoal em homens portadores de esquizofrenia em tratamento com haloperidol ou clozapina. Interação em Psicologia, 10(1), 151-156. doi: 10.5380/psi.v10i1.5708

Keefe, R. S. R., \& Eesley, C. E. (2012). Déficits neurocognitivos. In J. A. Lieberman, T. S. Stroup, \& D. O. Perkins, Fundamentos da esquizofrenia (pp. 89-108). Porto Alegre, RS: Artmed.

Kimhy, D., Corcoran, C., Harkavy-Friedman, J. M., Ritzler, B., Javitt, D. C., \& Malaspina, D. (2007). Visual form perception: A comparison of individuals at high risk for psychosis, recent onset schizophrenia and chronic schizophrenia. Schizophrenia Research, 97(1-3), 25-34. doi: 10.1016/j.schres.2007.08.022

Londere, M. D. B., Massuda, R., \& Gama, C. S. (2015). Neurobiologia da esquizofrenia. In A. E. Nardi, J. Quevedo, \& A. G. Silva (Eds.), Esquizofrenia: Teoria e Clínica. Porto Alegre, RS: Artmed.

Lukasova, K., Zanin, L. L., Chucre, M. V., Macedo, G. C., \& Macedo, E. C. (2010). Analysis of exploratory eye movements in patients with schizophrenia during scanning of projective tests' figures. Jornal Brasileiro de Psiquiatria, 59(2), 119-125. doi: 10.1590/S0047-20852010000200007

Malloy-Diniz, L., Paula, J. J., \& Sedó, M. (2014). Neurospicologia das funções executivas e da atenção (pp. 115-138). In D. Fuentes, Neuropsicologia: Teoria e prática. Porto Alegre, RS: Artmed.

Meyer, G. J., Viglione, D. J., MIhura, J. L., Erard, R. E., \& Erdberg, P. (2011). Rorschach Perfomance Assessment System: Administration, coding, interpretation, and technical manual. Toledo, Ohio: Rorschach Performance Assessment System.

Miguel, F. K. (2014). Mitos e verdades no ensino de técnicas projetivas. PsicoUSF, 19(1), 97-106. doi: 10.1590/1413-82712014019003015

Moore, R. C., Viglione, D. J., Rosenfarb, I. S., Patterson, T. L., \& Mausbach, B. T. (2013). Rorschach measures of cognition relate to everyday and social functioning in schizophrenia. Psychological Assessment, 25(1), 253-263. doi: $10.1037 / \mathrm{a} 0030546$

Murray, H. A. (2005). TAT: Teste de apercepção temática. São Paulo, SP: Casa do Psicólogo. 
Noto, C., Ortiz, B. B., \& Honda, P. H. S. (2015). Esquizofrenia resistente ao tratamento. In A. F. Carvalho, A. E. Nardi, \& J. Quevedo (Eds.) Transtornos psiquiátricos resistentes ao tratamento: Diagnóstico e manejo. Porto Alegre, RS: Artmed.

Organização Mundial da Saúde. (1993). Classificação de transtornos mentais e de comportamento da CID-10: Descrições clínicas e diretrizes diagnósticas. Porto Alegre, RS: Artes Médicas

Ortiz, B. \& Lacaz, F. S. (2012). Esquizofrenia: Diagnóstico e dimensões clínicas. In C. S. Noto \& R. A. Bressan (Eds.) Esquizofrenia: Avanços no tratamento multidisciplinar. Porto Alegre, RS: Artmed.

Pasian, S. R. (2000). O psicodiagnóstico de Rorschach em adultos: Atlas, normas e reflexões. São Paulo, SP: Casa do Psicólogo.

Pasian, S. R. \& Loureio, S. R. (2010). Reflexões sobre princípios e padrões normativos do Rorschach. In S. R. Pasian (Ed.), Avanços do Rorschach no Brasil. São Paulo, SP: Casa do Psicólogo.

Pegoraro, L. F. L., Ceará, A. T., \& Fuentes, D. (2014). Neuropsicologia das psicoses (pp. 215-222). In D. Fuentes, Neuropsicologia: Teoria e prática. Porto Alegre, RS: Artmed.

Pereira, C. \& Chehter, E. Z. (2011). Associações entre impulsividade, compulsão alimentar e obesidade em adolescentes. Arquivos Brasileiros de Psicologia, 63(3), 16-30.

Pereira, R. S. (2011). Transtornos dissociativo e histriônico: contribuições da avaliação psicodiagnóstica. (Monografia). Universidade de São Paulo, São Paulo.

Primi, R. (2010). Avaliação psicológica no Brasil: Fundamentos, situação atual e direções para o futuro. Psicologia: Teoria e Pesquisa, 26(especial), 25-35. doi: 10.1590/S0102-37722010000500003

Rausch de Traubenberg, N. (1975). A prática do Rorschach. São Paulo, SP: Cultrix.

Resende, A. C., \& Argimon, I. I. L. (2012). A técnica de Rorschach e os critérios da CID-10 para o diagnóstico da esquizofrenia. Psicologia: Reflexão e Crítica, 25(3), 422-434. doi: 10.1590/S0102-79722012000300002

Roisko, R., Wahlberg, K. E., Hakko, H., Wynne, L. \& Tienari, P. (2011). Communication deviance in parents of families with adoptees at a high or low risk of schizoprenia-spectrum disordes and its associations with attributes of the adoptee and the adoptive parents. Psychiatry Research, 185, 66-71. doi: 10.1016/j.psychres.2010.04.018

Rosenbaum, B., Andersen, P. B., Knudsen, P. B. \& Lorentzen, P. (2012). Rorschach inkblot method data at baseline and after 2 years treatment of consecutively admitted patients with first-episode schizophrenia. Nordic Journal of Psychiatry, 66(2), 79-85. doi: 10.3109/08039488.2011.595500 
Rueda, F. J. M., \& Lamounier, R. (2006). O psicodiagnóstico miocinético em motoristas: diferenças por gênero, escolaridade e idade. Avaliação Psicológica, 5(2), 179-187.

Salim, J. (1987). Noções de psicofarmacologia na prática. São Paulo, SP: EPU: EDUC.

Santos, M. A. (2000). A representação de si na esquizofrenia através do psicodiagnóstico de Rorschach. Paidéia, 10(19), 67-81. doi: 10.1590/S0103-863X2000000200009

Santos, M. A., \& Vaz, C. E. (2006). Representação de objeto e organização psíquica: Integração dinâmica dos dados do Rorschach. Psico, 37(3), 249261.

Senden, D. A., Lopes, R. M. F., Lopes do Nascimento, R. F. L., Terroso, L. B., \& Argimon, I. I. L. (2013). Análise da personalidade de profissionais da área da saúde: Um estudo pelo teste Palográfico. Aletheia, 42, 62-70.

Sisto, F. F. (2010). Uma análise das evidências de validade brasileiras do PMK. Psico-USF, 15(2), 141-149. doi: 10.1590/S1413-82712010000200002

Stroup, T. S., Marder, S. R., \& Lieberman, J. A. (2012). Tratamento farmacológico (pp. 189-224). In J. A. Lieberman, T. S. Stroup, \& D. O. Perkins (Eds.), Fundamentos da esquizofrenia. Porto Alegre, RS: Artmed.

Tardivo, R. C. (2003). Casa-Árvore-Pessoa (HTP): Manual e guia de interpretação. São Paulo, SP: Vetor.

Urbina, S. (2007). Fundamentos da testagem psicológica. Porto Alegre, RS: Artmed.

Valença, A. M., \& Nardi, A. E. (2015). Histórico do conceito de esquizofrenia. In A. E. Nardi, J. Quevedo, \& A. G. Silva, (Eds.), Esquizofrenia: Teoria e Clínica (pp. 17-24). Porto Alegre: Artmed.

Vasconcelos, A. G., Do Nascimento, E., \& Sampaio, J. R. (2011). PMK: Validade preditiva do PMK em relação à presença de sintomas psicopatológicos. Psico-USF, 16(2), 143-149. doi: 10.1590/S1413-82712011000200003

Vasconcelos, A. G., Sampaio, J. R., \& Do Nascimento, E. (2013). PMK: Medidas válidas para a predição do desempenho no trabalho? Psicologia: Reflexão e Crítica, 26(2), 251-260. doi: 10.1590/S0102-79722013000200005

Vaz, C. E. (1997). O Rorschach: Teoria e desempenho. São Paulo, SP: Manole Ltda.

Vieira, C., Fay, E. S. M., \& Neiva-Silva, L. (2007). Avaliação psicológica, neuropsicológica e recursos em neuroimagem: Novas perspectivas em saúde mental. Aletheia, 26, 181-195.

Vieira, P. G., \& Villemor-Amaral, A. E. (2015). Evidências de validade do Rorschach Performance Assessment System no diagnóstico da esquizofrenia. Avaliação Psicológica, 14(1), 53-62. doi: 10.15689/ap.2015.1401.06 
Villemor-Amaral, A. E. (2012). As pirâmides coloridas de Pfister. São Paulo, SP: Casa do Psicólogo.

Villemor-Amaral, A. E., \& Machado, M. A. S. (2011). Indicadores de depressão do Zulliger no Sistema Compreensivo (ZSC). Paidéia, 21(48), 21-27. doi: $10.1590 / \mathrm{S} 0103-863 \times 2011000100004$

Villemor-Amaral, A. E., \& Primi, R. (2012). Teste de Zulliger no sistema compreensivo, ZSC: Forma individual. São Paulo, SP: Casa do Psicólogo.

Villemor-Amaral, A. E., Primi, R., Franco, R. R. C., Farah, F. H. Z., Cardoso, L. M., \& Silva, T. C. (2005). O teste de Pfister e sua contribuição para o diagnóstico da esquizofrenia. Revista do Departamento de Psicologia - UFF, 17(2), 89-98. doi: 10.1590/S0104-80232005000200008

Zimmer, M., Jou, G. I., Sebastiany, C. M., Guimarães, E. R., Boechat, L. C., Soares, T., \& Belmonte-de-Abreu, P. S. (2008). Avaliação neuropsicológica na esquizofrenia: revisão sistemática. Revista de Psiquiatria, 30(1 Supl.). doi: 10.1590/S0101-81082008000200005

Sobre os autores

Ana Carolina Zuanazzi é psicóloga pela Universidade Estadual de Londrina e mestre em Psicologia Clínica pelo Instituto de Psicologia da Universidade de São Paulo. anacarolina.zf@gmail.com

Rafaela Larsen Ribeiro é graduada em Ciências Biológicas pela Universidade Federal do Paraná, mestre em Farmacologia pela Universidade Federal de Santa Catarina e doutora em Psicobiologia pela Universidade Federal de São Paulo. profarafaelaribeiro@gmail.com

Recebido em: 09/09/2015 Revisado em: 14/10/2015 Aceito em: 16/11/2015 